Ein Jahr UN-Bildungsdekade

\section{Ein Zwischenfazit zur Hochschullehre}

Die UN-Dekade Bildung für nachhaltige Entwicklung ist in ihr zweites Jahr gegangen. Ein langer Umsetzungsprozess liegt noch vor ihr, möchte man meinen. Aber wichtige Weichenstellungen müssen bereits jetzt vorgenommen werden - gerade in traditionell eher verfestigten Strukturen wie denen der Hochschule. Von Bernhard Schowe-von der Brelie

$\mathbf{F}_{\mathrm{v}}^{\mathrm{u}}$ ür die Jahre 2005 bis 2014 haben die Vereinten Nationen die Dekade „Bildung für Nachhaltige Entwicklung“ ausgerufen, für deren nationale Ausgestaltung die deutsche UNESCO-Kommission ein eigenes Nationalkomitee beauftragt hat. Dessen Aktivitäten, die über ein Expertenpanel und Arbeitsgruppen im Kontakt mit der (Fach-)Öffentlichkeit gesteuert werden, weisen derzeit einen deutlichen Fokus auf den schulischen und vorschulischen Bildungsbereich auf.

Mit dem Ziel, das Nachhaltigkeitskonzept als präanalytische Vision möglichst frühzeitig zu vermitteln, mag dieser Schwerpunkt angemessen sein. Richtigerweise aber hat das Nationalkomitee auch die sinnvolle Verknüpfung aller Bildungsebenen als Voraussetzung für eine Nachhaltigkeits-Bildung als gesellschaftlichen, lebensweltlichen wie lebenslangen Prozess erkannt und entsprechende Ziele und Maßnahmen formuliert.

\section{Hochschulen sind die zentralen Akteure}

Damit sind auch und vor allem Hochschulen aufgefordert, sich aktiv an der Bildungsdekade zu beteiligen. Handlungsfelder lägen in vielfältigen Bereichen, die weit über das traditionelle Bildungs-Kerngeschäft Lehre hinausgehen: in der engen Verzahnung mit der Nachhaltigkeitsforschung, in der Mitwirkung in (regionalen) Bildungs- und Innovationsnetzwerken als außerschulische Lernorte, im öffentlichen Dialog oder in der nachhal- tigen Ausgestaltung der Lebenswelt Hochschule.

Offensichtlich ist der Impuls durch die UN aber nicht oder zumindest noch nicht genutzt. Unter den 288 bis Ende 2009 ausgezeichneten Dekadeprojekten finden sich gerade einmal zwölf mit Hochschulbeteiligung - ohne dass dabei stets die spezifischen Potenziale von Hochschulen aufgegriffen werden.

Bildungspolitik, zumal Hochschulpolitik, ist Ländersache. Nur wenige Bundesländer haben der Bildungsdekade aber bisher eigene Impulse, noch weniger im Hochschulbereich, gegeben. Positiv hervorzuheben sind allein Nordrhein-Westfalen und die vier Länder der „Norddeutschen Partnerschaft“ - wenn auch bislang leider ohne sichtbare Folgeprozesse.

Die deutschen Hochschulen sind sicherlich gerade das Gegenteil einer homogenen Masse, die sich in eine bestimmte Richtung bewegen ließe. Umso mehr fehlt eine bundesweit wirkende Instanz, die die Einzelmaßnahmen und -initiativen an den Hochschulen vernetzt und gegebenenfalls die notwendigen Rahmenbedingungen ableitet und politisch durchsetzt: HRK und KMK, die als Netzwerk geplante, aber noch immer nicht umgesetzte „Hochschulplattform UNI21“ und der neu eingerichtete UNESCO Chair in Higher Education for Sustainable Development an der Universität Lüneburg stehen in der Pflicht, dem Dekadeprozess im kommenden Jahr neue Impulse zu geben.
Der Zeitpunkt ist günstig. Bologna, Öffnung, Ökonomisierung, Profilbildung und Wettbewerb setzen die Hochschulen ohnehin unter Reformdruck. Die Umstellung auf gestufte Studiengänge birgt die große, bislang kaum genutzte Chance, inter- und transdisziplinäre Elemente für eine Nachhaltigkeitsbildung in den neuen Curricula zu verankern.

\section{Profilbildung notwendig}

Eine echte Profilbildung der Hochschulen hinsichtlich einer Nachhaltigkeits-Lehre muss zudem verstärkt als ein entscheidender Beitrag für eine zukunftsorientierte, auf technische und gesellschaftliche Innovation gründende „exzellente Spitzenbildung“ im internationalen Kontext verstanden werden. Hier ist sicherlich noch dem Vorurteil einer ausschließlichen Öko-Lehre vermehrt zu begegnen.

Die Bildungsdekade ist in ihrem zweiten Jahr in den Hochschulen noch nicht richtig angekommen. Umso wichtiger ist es, diesen meist in einer langen Tradition stehenden, oftmals schwerfälligen Institutionen frühzeitig die richtigen Impulse zu geben. Gute Beispiele an einzelnen Hochschulen gibt es viele. Doch diese Leuchtturmprojekte erhellen weder die gesamte Republik, noch bieten sie umfassende Orientierung für alle.

Es ist höchste Zeit, ihre Aktivitäten zu vernetzen und ihnen im Rahmen der Bildungsdekade eine gemeinsame Richtung zu geben. So könnten Hochschulen „kritische Instanz und Motor für dauerhaftes gesellschaftliches Wohlergehen“ werden, wie es die Dekade-Arbeitsgruppe „Hochschulen - Forschung, Lehre, Service“ in ihrem ersten Papier fordert. Das Potenzial dazu ist vorhanden!

I AUTOR + KONTAKT Bernhard Schowe-von der Brelie ist wissenschaftlicher Mitarbeiter der Hochschul-Informations-System $\mathrm{GmbH}$ sowie Vorstandsmitglied der Vereinigung für ökologische Wirtschaftsforschung e.V. (VÖW).

VÖW, Potsdamer Straße 105, 10785 Berlin. E-Mail: bernhard.schowe@voew.de, www.voew.de. 
(c) 20I0 Authors; licensee IÖW and oekom verlag. This is an article distributed under the terms of the Creative Commons Attribution Non-Commercial No Derivates License (http://creativecommons.org/licenses/by-nc-nd/3.o/), which permits unrestricted use, distribution, and reproduction in any medium, provided the original work is properly cited. 